\title{
Bayesian Probabilistic Framework for Damage Identification of Steel Truss Bridges under Joint Uncertainties
}

\author{
Wei Zheng and Yi Yu \\ Department of Civil and Environmental Engineering, Jackson State University, 1400 J. R. Lynch Street, P.O. Box 17068, Jackson, \\ MS 39217, USA \\ Correspondence should be addressed to Wei Zheng; wei.zheng@jsums.edu
}

Received 25 March 2013; Accepted 18 July 2013

Academic Editor: Andreas Kappos

Copyright (C) 2013 W. Zheng and Y. Yu. This is an open access article distributed under the Creative Commons Attribution License, which permits unrestricted use, distribution, and reproduction in any medium, provided the original work is properly cited.

\begin{abstract}
The vibration-based structural health monitoring has been traditionally implemented through the deterministic approach that relies on a single model to identify model parameters that represent damages. When such approach is applied for truss bridges, truss joints are usually modeled as either simple hinges or rigid connections. The former could lead to model uncertainties due to the discrepancy between physical configurations and their mathematical models, while the latter could induce model parameter uncertainties due to difficulty in obtaining accurate model parameters of complex joint details. This paper is to present a new perspective for addressing uncertainties associated with truss joint configurations in damage identification based on Bayesian probabilistic model updating and model class selection. A new sampling method of the transitional Markov chain Monte Carlo is incorporated with the structure's finite element model for implementing the approach to damage identification of truss structures. This method can not only draw samples which approximate the updated probability distributions of uncertain model parameters but also provide model evidence that quantify probabilities of uncertain model classes. The proposed probabilistic framework and its applicability for addressing joint uncertainties are illustrated and examined with an application example. Future research directions in this field are discussed.
\end{abstract}

\section{Introduction}

Steel truss bridges are commonly used in the highway system. Those truss bridges are typically composed of slender steel members connected at truss joints. The truss joints may take various types or configurations. During their longterm operations, steel truss bridges may become deteriorated (such as development of fatigue cracks and corrosions) due to increased volumes of traffic and adverse environment impacts. Such deterioration or damage could pose serious threats to the safe operation of bridges if its development cannot be identified in a timely manner. Researchers have explored various approaches for effectively detecting the development of deterioration or damages of truss structures at their early stages through implementing vibration-based structural health monitoring (SHM), which typically relies on vibration measurements and structural models to identify model parameters that represent extents and locations of damages.
Gao et al. $[1,2]$ had proposed an approach of diagnosis locating vector (DLV) for damage identification of steel truss structures by using distributed computing strategy (DCS). They verified this approach by using computational simulation of damage identification of a simple 14-bay planar truss structure. Spencer et al. [3] and Ji et al. [4] further validated the effectiveness of the above DCS method through experiments on a truss model and a two-storey steel bracedframe model. Sohn [5] introduced an approach of loaddependent Ritz vectors as an alternative approach to identify damages in an eight-bay truss structure. In the above studies, truss joints were simply modeled as hinges in the structure model that was used for the damage identification. The impact of types of the joint model (e.g., hinged, semirigid, or rigid) on outcomes of the damage diagnosis of truss structures was not explicitly addressed.

Some researchers, on the other hand, have attempted to explicitly consider the complexity of truss joint configurations in their models for damage identification of truss 
structures. Jones et al. [6] and Lewitt et al. [7] considered that the true behavior of joints is between the fully-rigid joints and the perfectly-pinned joints and includes somewhat nonlinear behaviors. By using data from field monitoring, Li et al. [8] had developed a multiscale bridge model that includes the detailed model of the joint configurations. They adopted this model to identify local deterioration of two typical weld connections in a steel truss bridge. Based on the global dynamic response data, Zhu and He [9] adopted a detailed finite element model of the bolted connection for detecting damages of a three-bay steel frame structure. Yun [10] developed the Steady-state genetic algorithm (SSGA) for damage identification of truss structures. They adopted detailed joint models in the truss finite element model and demonstrated the performance of their proposed approach through a 14-bay planar truss. Law et al. [11] presented a model updating method for a superelement model, in which joints were modeled as the semirigid joints with specified stiffness. They validated their approach to identifying the damage of joints of a three-dimensional 10-bay cantilevered truss structure.

Among two types of strategies for modeling joints of steel truss structures adopted in the previous research, one adopts oversimplified models of truss joint that could lead to model uncertainties due to the discrepancy between joint physical configuration and its model representation; the other could induce more uncertainties associated with model parameters due to difficulties in obtaining accurate model parameters of complex joint configurations. Both strategies could cause the deviation of the identified model parameters that represent damages from their true physical damages and lead to masking real damages or triggering false damage alarms. Even though progresses have been made in vibration-based structural health monitoring of the truss or truss-like structures, most of existing methods rely on deterministic methods that cannot evaluate the impacts of uncertainties associated with the model of joint configurations on outcomes of damage identification. Because the in situ measurement data is always limited for a complex truss structure with many unknown or uncertain model parameters, deterministic approaches to damage identification often yield ill conditions (unidentifiable solution) or nonuniqueness (multiple solution) and cannot address inherent uncertainties associated with the structural model and model parameters.

With those uncertainties present, monitoring and assessing damages of steel truss bridges may better be conducted within a probabilistic framework. The recently resurgent Bayesian inference could provide an applicable computational framework for robustly addressing uncertainties associated with models and model parameters in damage identification. Within this framework, uncertainties associated with structural models are represented by the probability of model among competing candidate models, and unknown damage status of structural members is presented by the probability distributions of model parameters. The probabilities of competing candidate models and the probability distributions of uncertain model parameters can be first assumed based on prior knowledge and engineering judgment and then updated based on Bayes' theorem by using available in situ measurement data. These updated model probabilities and probability distributions of model parameters not only identify the most suitable model among competing candidate models and estimate the most suitable model parameters that correspond to the damage location and extent but also quantify uncertainties in such identification and estimation, which are essential for assessing the reliability-based performance of the system for decision making. For example, the quantified uncertainties obtained from probabilistic inference can be used to make a statement such as that the probability of a specific truss member damaged with stiffness reduction ranging from $20 \%$ to $30 \%$ is $90 \%$.

The general framework of Bayesian inference for structural model updating and damage identification was presented by Beck and his associates in the 90s [12]. Subsequent research has shown the effectiveness of Bayesian probabilistic inference in assessing uncertainties in structural model updating and damage identification. Yuen and Katafygiotis [13] present a time-domain Bayesian inference approach to update uncertain modal parameters in a four-bay simple truss model by using measured time histories of ambient response. Beck and Yuen [14] demonstrated Bayesian model selection for system identification based on dynamic response of nonlinear oscillator among competing candidate models. Chen and Feng [15] illustrated the effectiveness of Bayesian inference for consistently estimating the system parameters and their uncertainties through two simulation examples and one shake table test. Ntotsios et al. [16] adopted Bayesian inference to bridge damage identification by using acceleration measurements and demonstrated its effectiveness by using simulated measurements of a prototype bridge, as well as using experimental vibration data from a laboratory small-scaled bridge model. However, the application of the Bayesian probabilistic framework has not been fully explored for addressing uncertainties associated with models of truss joint configurations that are encountered in monitoring and assessing damages of steel truss bridges.

This paper presents probabilistic computational framework for identifying structural damage of the truss members under uncertainties associated with modeling joint configurations by using vibration measurements. The framework integrates the advanced sampling algorithm of the transitional Markov chain Monte Carlo with structure's finite element model. It can be used to effectively draw statistical samples that approximate the updated probabilistic distributions of uncertain model parameters and provide the model evidence that can be used to quantify probabilities of uncertain model classes. The paper in the subsequent sections is organized as follows. Firstly, the theoretic framework of Bayesian inference for damage identification is outlined. Secondly, computational procedures that integrate the structural model with advanced sampling algorithm are presented. Thirdly, an application of the proposed framework and its effectiveness are illustrated and examined through numerical simulation of damage identification of nine-bay three-dimensional steel truss model. Finally, future research directions in this field are discussed. 


\section{Theoretical and Computational Framework}

2.1. Damages Identification through Model Updating. Vibration measurements have been used in SHM for identifying structural damages of engineering systems. The vibrationbased SHM approach is based on the premises that structural dynamic characteristics are functions of structural properties and boundary conditions, and can be reflected in the vibration responses of the structures under external dynamic excitations. As damages cause the change of structural properties, the measured vibration responses from the structure will also alternate. The process of the model updating or calibration is to determine the most suitable model parameters, which are related to damage status, structural properties, or boundary conditions, through minimizing the difference between the predicted structural response from the structural model and the measured structural response from sensor networks installed in the structure.

In model updating process, uncertain model parameters that need to be calibrated are unknown or cannot be predetermined accurately. In the context of damage identification, structural damages are usually parameterized in terms of the change of stiffness of structure members between damaged structures and undamaged structures, because the damage would cause the reduction of stiffness of an individual structural member or a group of structural members (i.e., a substructure). Thus, structural damages can be parameterized mathematically into the global stiffness matrix of a finite element model as follows:

$$
\mathbf{K}(\boldsymbol{\alpha})=\mathbf{K}_{0}+\sum_{i=1}^{N} \alpha_{i} \mathbf{K}_{i},
$$

where $\mathbf{K}(\boldsymbol{\alpha})$ is the global stiffness matrix of a finite element model; $\mathbf{K}_{0}$ is the part of the global stiffness matrix contributed from the structural members which are assumed to be undamaged; $\mathbf{K}_{i}$ is the part of the global stiffness matrix contributed from the $i$ th structural members which is suspected to be damaged; $\alpha_{i}$ is the stiffness reduction factor that indicates the damage extent and location in the structure for example; for $\alpha_{i}=1$ it represents no damage, and for $\alpha_{i}=0$ it represents complete loss of stiffness or resistance.

In another similar formulation of damage identification, when measurement data are available for both before and after damage occurrence, $\mathbf{K}_{i}$ in the above formula can be considered as initial estimated stiffness contributed from $i$ th structural members, and the $\alpha_{i}^{u d}$ and $\alpha_{i}^{d a}$ are the stiffness factors for both before and after damages occurrence and can be obtained for both before and after damages occurrence, respectively. The change of $\Delta \alpha_{i}=\alpha_{i}^{u d}-\alpha_{i}^{d a}$ before and after damages can be used to reveal the damage extents and locations.

2.2. Bayesian Model Updating and Model Class Selection. If there are uncertainties associated with model parameters for a specific model of the system $M_{j}$, uncertain model parameters can be denoted as a vector $\boldsymbol{\alpha}$, where the dimension of $\boldsymbol{\alpha}$ is $N_{\alpha}$, and may take different values. As a result, the structural model $M_{j}$ would correspond to a class of models for different model parameter vector $\boldsymbol{\alpha}$. Thus, in the Bayesian inference framework, one specific type of structural model $M_{j}$ is usually referred to as a model class $M_{j}$. For a given specific value for $\boldsymbol{\alpha}$, the model $M_{j}$ and $\boldsymbol{\alpha}$ describe the relation between the model input vector $\mathbf{Z}$ and its output vector $\mathbf{X}$; that is, $\mathbf{X}=q_{i}\left(\boldsymbol{\alpha}, \mathbf{Z}, M_{j}\right)$. When there are uncertainties associated with the structural model of a specific structure system, the structural model class $M$ could possibly take different forms. As a result, classes of multiple competing candidate models denoted as $\mathbf{M}=\left\{M_{j}: j=1,2, \ldots, N_{M}\right\}$ may be considered for the structure system.

Bayesian inference essentially provides a probabilistic computational framework based on Bayes' theorem to quantify uncertainties associated with model parameters and model classes by specifying the probability density function (PDF) (or probabilistic distribution) of uncertain model parameters and relative model probabilities of competing candidate model classes [14, 17, 18]. The formulation of Bayesian inference can be applied in the time domain or in the spectrum domain for vibration-based structural health monitoring and damage identification. For obtaining details in this regard, readers may refer to $[14,17]$. This paper adopts Bayesian model updating and model selection in the time domain $[14,18]$, which is briefly outlined as follows.

The key for applying Bayes' theorem to update the probabilistic distributions of uncertain models and their parameters using the measurement data is to create a probability model $P$ with a model parameter vector $\boldsymbol{\theta}$ or the likelihood function $p\left(\mathbf{D} \mid \boldsymbol{\theta}, M_{j}\right)$, which defines the likelihood of getting the measurement data $\mathbf{D}$ for a given parameter vector $\boldsymbol{\theta}$ and a structural model $M_{j}$, where the probability mode parameter vector $\boldsymbol{\theta}$ contains the structural model parameter vector $\boldsymbol{\alpha}$ and other parameters that describe probabilistic characteristics of the probability mode and are defined subsequently. The likelihood function $p\left(\mathbf{D} \mid \boldsymbol{\theta}, M_{j}\right)$ can be established based on the probability density function of the prediction error, that is, the difference between the measured system outputs from in situ sensors deployed at selected locations and the predicted system outputs from the structural model with given model parameter $\boldsymbol{\alpha}$ contained in the parameter vector $\boldsymbol{\theta}[18]$.

If the available input-output data $\mathbf{D}$ contains the measured input vector $\mathbf{Z}_{\mathbf{n}}$ and output (or response) vector $\mathbf{Y}_{\mathbf{n}}$ for $N_{d}$ of selected degree of freedom (DOF) of the system, which corresponds to measurement locations at different times, $t=$ $n \cdot \Delta t$, where $n=1,2, \ldots, N_{t}$, and $N_{t}$ is the total number of time series of measurement, then the prediction error vector, $\mathbf{e}_{\mathrm{n}}$, at the time $t=n \cdot \Delta t$ and at the selected DOF of the structural system for a given structural model $M_{j}$ and its parameter vector $\boldsymbol{\alpha}$ can be expressed as

$$
\mathbf{e}_{\mathbf{n}}=\mathbf{Y}_{\mathbf{n}}-L_{0} \cdot q_{j}\left(\boldsymbol{\alpha}, \mathbf{Z}_{\mathbf{n}}, M_{j}\right),
$$

where $q_{j}\left(\boldsymbol{\alpha}, \mathbf{Z}_{\mathbf{n}}, M_{j}\right)$ is a vector of the predicted outputs at all degrees of freedom (DOF) of the structural model $M_{j}$ with given structural model parameter vector $\boldsymbol{\alpha}$ and input vector $\mathbf{Z}_{\mathbf{n}}$ at time $t=n \cdot \Delta t ; \mathbf{L}_{0}$ is the selection matrix with only nonzero element equal to 1 in each row for converting the output vector $q_{j}\left(\boldsymbol{\alpha}, \mathbf{Z}_{\mathbf{n}}, M_{j}\right)$ at all DOF of the structural 
model $M_{j}$ into the output vector at the measured DOF, so that $L_{0} \cdot q_{j}\left(\boldsymbol{\alpha}, \mathbf{Z}_{\mathbf{n}}, M_{j}\right)$ is the vector of the predicted system outputs at $N_{d}$ of measured degree of freedom (DOF) of the model $M ; \mathbf{e}_{\mathbf{n}}, \mathbf{Y}_{\mathbf{n}}$, and $L_{0} \cdot q_{j}\left(\boldsymbol{\alpha}, \mathbf{Z}_{\mathbf{n}}, M_{j}\right)$ are vectors with $N_{d}$ dimensions.

It is usually assumed that the prediction error vector $\mathbf{e}_{\mathbf{n}}$ is a zero-mean stationary normally-distributed stochastic process with a standard deviation of $\sigma$, which is equal for all DOF of the model. It is also assumed that the prediction errors at different times and at different locations are independent. Then, the likelihood function $p\left(\mathbf{D} \mid \boldsymbol{\theta}, M_{j}\right)$ can be represented as the probability density function of joint distribution of multiple independent normal distributed statistical variables (total number is $N_{t} \cdot N_{d}$ ) as follows:

$$
\begin{aligned}
p\left(\mathbf{D} \mid \boldsymbol{\theta}, M_{j}\right) \\
=\frac{1}{\sqrt{2 \pi} \sigma^{N_{t} \cdot N_{d}}} \\
\quad\left[-\frac{1}{2 \cdot \sigma^{2}} \sum_{n=1}^{N_{t}} \sum_{j=1}^{N_{d}}\left[Y_{n}^{(k)}-\left\{L_{0} \cdot q_{j}\left(\boldsymbol{\alpha}, \mathbf{Z}_{\mathbf{n}}, M_{j}\right)\right\}^{(k)}\right]^{2}\right],
\end{aligned}
$$

where $\boldsymbol{\theta}=\left\{\boldsymbol{\alpha}^{T}, \sigma\right\}^{T}$ is the uncertain parameter vector of the probability model $P$ as mentioned before and needs to be updated through Bayes' theorem with measurement data; $Y_{n}^{(k)}$ is the $k$ th component of the vector $Y_{n}$ at the time $t=n \cdot \Delta t$; and $\left\{L_{0} \cdot q_{j}\left(\boldsymbol{\alpha}, \mathbf{Z}_{\mathbf{n}}, M_{j}\right)\right\}^{(k)}$ is the $k$ th component of the vector $L_{0} \cdot q_{j}\left(\boldsymbol{\alpha}, \mathbf{Z}_{\mathbf{n}}, M_{j}\right)$ at the time $t=n \cdot \Delta t$.

For the specific candidate structural model class $M_{j}$, the prior probability density function (PDF) of model parameters $p\left(\begin{array}{lll}\boldsymbol{\theta} & M_{j}\end{array}\right)$ can usually be preassumed based on prior knowledge or engineering judgment on the statistical distribution of parameters $\boldsymbol{\theta}$; for example, $p\left(\boldsymbol{\theta} \mid M_{j}\right)$ can be a normal distribution or uniform distribution between specific values. Once the measurement data $\mathbf{D}$ are available from instrumentation installed in the structure system, the posterior PDF $p\left(\boldsymbol{\theta} \mid \mathrm{D}, M_{j}\right)$ of parameters can be obtained based on Bayes' theorem as follows:

$$
p\left(\boldsymbol{\theta} \mid \mathbf{D}, M_{j}\right)=\frac{p\left(\mathbf{D} \mid \boldsymbol{\theta}, M_{j}\right) \cdot p\left(\boldsymbol{\theta} \mid M_{j}\right)}{p\left(\mathbf{D} \mid M_{j}\right)},
$$

where $p\left(\boldsymbol{\theta} \mid M_{j}\right)$ is the prior PDF and can be preassumed; $p\left(\mathbf{D} \mid \boldsymbol{\theta}, M_{j}\right)$ is the likelihood function as defined in (3); $p(\mathbf{D} \mid$ $M_{j}$ ) is called the model evidence (or the marginal likelihood function), actually is a normalization factor that makes the integral of the right side in (4) over all spaces of parameters $\boldsymbol{\theta}$ to be equal to 1 , and thus can be determined as follows:

$$
p\left(\mathbf{D} \mid M_{j}\right)=\int p\left(\mathbf{D} \mid \boldsymbol{\theta}, M_{j}\right) \cdot p\left(\boldsymbol{\theta} \mid M_{j}\right) d \boldsymbol{\theta} .
$$

When there are uncertainties associated with the structural model of a specific system, classes of multiple competing candidate structural models may be considered for the system and denoted by $\mathbf{M}=\left\{M_{j}: j=1,2, \ldots, N_{M}\right\}$. With the measurement data $\mathbf{D}$ available, Bayesian model class selection can be conducted to obtain the posterior probability $P\left(M_{j}\right.$ | $\mathbf{D}, \mathbf{M})$ of each model class $M_{j}$ in the model classes $\mathbf{M}$ based on Bayes' theorem as follows:

$$
P\left(M_{j} \mid \mathbf{D}, \mathbf{M}\right)=\frac{p\left(\mathbf{D} \mid M_{j}\right) \cdot P\left(M_{j} \mid \mathbf{M}\right)}{\sum_{j=1}^{N_{M}} p\left(\mathbf{D} \mid M_{j}\right) \cdot P\left(M_{j} \mid \mathbf{M}\right)},
$$

where $P\left(M_{j} \mid \mathbf{M}\right)$ is the prior probability of model class $M_{j}$ and can be taken to be $1 / N_{M}$ if it is reasonable to consider all model classes to be equally plausible; $N_{M}$ is the number of model classes; $p\left(\mathbf{D} \mid M_{j}\right)$ is the model evidence for model $M_{j}$ as defined in (5).

The model class with the largest posterior probability $P\left(M_{j} \mid \mathbf{D}, \mathbf{M}\right)$ will be the most plausible model among the model classes $\mathbf{M}$ and can be used to represent the system behaviors. If needed, the combination of several of the most suitable models weighted with their probability can be used to represent the system behaviors based on Bayesian model class averaging [18].

\section{Probabilistic Simulation}

The posterior PDF of model parameters as defined in (4) usually cannot be obtained explicitly. This is not only because that the posterior PDF may have a complicated format, but also because that the calculation of posterior PDF needs assessment of the model evidence $p\left(\mathbf{D} \mid M_{j}\right)$ and requires the evaluation of an integral over the space of model parameters as indicated in (6), which cannot be evaluated analytically due to higher dimensions of the parameter vector $\boldsymbol{\theta}[14,17,18]$. Particularly for structural model updating or damage identification, the evaluation of the likelihood function also needs the calculation of the predicted response from the structural finite element model. This makes analytical evaluation of the posterior PDF of model parameters impossible. Thus, the posterior PDF of uncertain model parameters defined in (4) is often evaluated and represented alternatively by using statistical samples drawn from posterior probability density functions (PDFs) through probabilistic simulation.

One commonly-implemented probabilistic simulation for drawing samples from the target PDF is the Markov Chain Monte Carlo (MCMC) algorithm proposed by Hastings [20, 21]. Based on well-defined criteria, the MCMC algorithm creates a chain of samples whose statistical distribution can approximate the target PDF by either accepting or rejecting the proposed samples from the spaces of parameters of interest. For detailed description of MCMC, the reader can refer to $[20,21]$. However, the standard MCMC algorithm may not be efficient when there is a very sharp peak or maxima or there are multiple peaks in the shape of the target PDF, because the drawn samples may become "stuck" in one local peak and cannot move to all other spaces of parameters of interest. Considering that the properties (or shapes) of the posterior PDFs are usually not known beforehand, Ching and Chen [19] had proposed the transitional Markov chain Monte Carlo (TMCMC) intended to be applied for all cases (e.g., very peaked PDFs and multiplepeaked PDFs). 
In the subsequent descriptions of TMCMC procedures, the subscript $j$ for a specific model class $M_{j}$ is dropped for convenience because the TMCMC is applied for each individual specific model class. The idea behind the TMCMC is to avoid the problem of sampling from difficult target PDFs but draw samples from a series of PDFs that are easier to sample and converge gradually to the target PDF. In addition, the TMCMC can draw samples from any probability distribution and require only that a function proportional to the target PDF to be calculated without knowing the normalization factor, such as the model evidence $f(\mathbf{D} \mid M)$ in (2). Furthermore, through drawing samples from series of intermediate PDFs, the model evidence $f(\mathbf{D} \mid M)$ can be obtained as a byproduct of drawing the samples of uncertain model parameters for evaluating the model probability. The TMCMC approach was established based on the technique initiated by Beck and $\mathrm{Au}$ [22] and includes consideration of a series of intermediate PDFs as follows [19]:

$$
\begin{array}{r}
p_{j}(\boldsymbol{\theta}) \propto p(\boldsymbol{\theta} \mid M) \cdot p(\mathbf{D} \mid \boldsymbol{\theta}, M)^{t_{j}}, \quad j=0, \ldots, m, \\
0=t_{0}<t_{1}<\cdots<t_{m}=1,
\end{array}
$$

where the operator $\propto$ denotes "is proportional to"; the index $j$ denotes the number of the stage or level of intermediate PDFs. It is assumed that the change between two adjacent intermediate PDFs is so small that the PDF of the current stage can be efficiently sampled from the PDF of its previous stage. For simplicity in the description below, the normalization constant $P(\mathbf{D} \mid M)$ as indicated in (4) is dropped in the following procedures, because those procedures are operated based on the ratio of PDFs at two different parameters and this normalization constant in the target PDF is canceled off.

Briefly speaking, the method of TMCMC uses series of intermediate PDFs, in which the first PDF in the sequence is the prior PDF $p_{0}(\boldsymbol{\theta})=p(\boldsymbol{\theta} \mid M)$ of model parameter when $j=0$ and $t_{j}=0$ in (6), and the last PDF in the sequence is the posterior $\mathrm{PDF} p_{m}(\boldsymbol{\theta})=p(\boldsymbol{\theta} \mid M) \cdot p(\mathbf{D} \mid \boldsymbol{\theta}, M)$ as defined in (4) when $j=m$ and $t_{m}=1$ in (7). By using the data from those intermediate PDFs, the model evidence $P(\mathbf{D} \mid M)$ defined in (5) can also be obtained for the selected model class $M$ and can be used to conduct Bayesian model class selection and model averaging [19]. For details of particular steps of algorithm in integrating TMCMC with Bayesian model updating and model class selection, readers can refer to [19]. The TMCMC algorithm [19] can be described as follows.

(1) Set the first intermediate PDF equal to the prior PDF, that is, $p_{o}(\boldsymbol{\theta})=p(\boldsymbol{\theta} \mid M)$. Select a uniformly distributed probability density function for the prior PDF $p(\boldsymbol{\theta} \mid M)$. Draw samples from the prior PDF $p(\boldsymbol{\theta} \mid M)$, and denote them by $\boldsymbol{\theta}_{k}^{0}, k=1, \ldots, N_{0}$, where $N_{0}$ is the number of samples of the unknown parameters at the stage $j=0$. Repeat the following steps total 2 to step 4 for $j=0,1, \ldots, \mathrm{m}-1$.

(2) Choose the tempering parameter $t_{j+1}$ for the next stage PDF to make sure that the coefficient of variance $(\mathrm{COV})$ of $p_{j+1}\left(\boldsymbol{\theta}_{k}^{j}\right) / p_{j}\left(\boldsymbol{\theta}_{k}^{j}\right)=p\left(\mathbf{D} \mid \boldsymbol{\theta}_{k}^{j}, M\right)^{t_{j+1}-t_{j}}$, $k=1, \ldots, N_{j}$, does not exceed some prescribed threshold, so that the next stage $\operatorname{PDF} p_{j+1}(\theta)$ is not much different from current $\operatorname{PDF} p_{j}(\boldsymbol{\theta})$, where $\boldsymbol{\theta}_{k}^{j}$, $k=1, \ldots, N_{j}$, are samples drawn from the PDF at the current stage.

(3) Calculat the plausibility weight $w\left(\boldsymbol{\theta}_{k}^{j}\right)=p(\mathbf{D} \mid$ $\left.\boldsymbol{\theta}_{k}^{j}, M\right)^{t_{j+1}-t_{j}}, k=1, \ldots, N_{j}$, and $S_{j}=\sum_{k=1}^{N_{j}} w\left(\boldsymbol{\theta}_{k}^{j}\right) / N_{j}$.

(4) Based on given $N_{j}$ samples $\boldsymbol{\theta}_{k}^{j}$ from the current stage PDF $p_{j}(\boldsymbol{\theta})$, generate $N_{j+1}$ samples $\boldsymbol{\theta}_{k}^{j+1}$ from the next stage PDF $p_{j+1}(\theta)$ by using following procedures. For $k=1, \ldots, N_{j+1}$, obtain $\boldsymbol{\theta}_{k}^{o}=\boldsymbol{\theta}_{l}^{j}$ with the probability $p=w\left(\boldsymbol{\theta}_{l}^{j}\right) / \sum_{n=1}^{N} w\left(\boldsymbol{\theta}_{n}^{j}\right)$ for $l=1,2, \ldots, N_{j}$. Then, draw $\boldsymbol{\theta}^{\mathrm{C}}$ (the superscript ${ }^{\mathrm{C}}$ denotes "candidate") from a normal distribution $N\left(\boldsymbol{\theta}_{k}^{o}, \sum_{j}\right)$, which has mean $\boldsymbol{\theta}_{k}^{o}$, and covariance matrix is $\sum_{j}$ that is defined by Ching and Chen [19]. Set $\boldsymbol{\theta}_{k}^{j+1}=\boldsymbol{\theta}^{\mathrm{C}}$ and $\boldsymbol{\theta}_{k}^{o}=\boldsymbol{\theta}^{\mathrm{C}}$ with the probability $p=p^{j+1}\left(\boldsymbol{\theta}^{\mathrm{C}}\right) / p^{j+1}\left(\boldsymbol{\theta}_{k}^{o}\right)$; otherwise, set $\boldsymbol{\theta}_{k}^{j+1}=\boldsymbol{\theta}_{k}^{o}$.

(5) Stop, if $t_{j+1}=t_{m}=1$ is reached.

\section{Computational Framework}

For the selected candidate structural model, the uncertain model parameters are selected as the parameters that need to be updated through Bayesian inference through drawing samples of parameters from the posterior PDF of parameters. While the candidate structural model is established by using finite element model, the TMCMC for sampling and Bayesian inference are coded and implemented in the MATLAB environment. To establish the computational framework, there is a need for the exchange of data between the finite element model and the MATLAB algorithm. To facilitate this data exchanging, the open-source program OpenSees is used to establish the finite element model for the structural system. OpenSees is the popular computational tool for studying dynamic responses of structures under the external excitations. It provides modular structures that allow users to develop and modify specific modules with relatively little dependence on other modules for the finite element analysis [23]. Even though written in $\mathrm{C}++$, the OpenSees allows users to use a special $\mathrm{Tcl}$ language to define the nodes, boundary conditions, and finite elements [23]. The Tcl language also can be used as a powerful script to control the execution and inputs/outputs of a large number of participating codes and allows exchanging of the inputs and outputs from OpenSees program into TMCMC and Bayesian inference algorithm in the MATLAB environment. In such a manner, the finite element model of the truss structures can be embedded in a probabilistic model and statistical simulation for drawing the samples that can be used to quantify uncertainties encountered in the damage identification. 


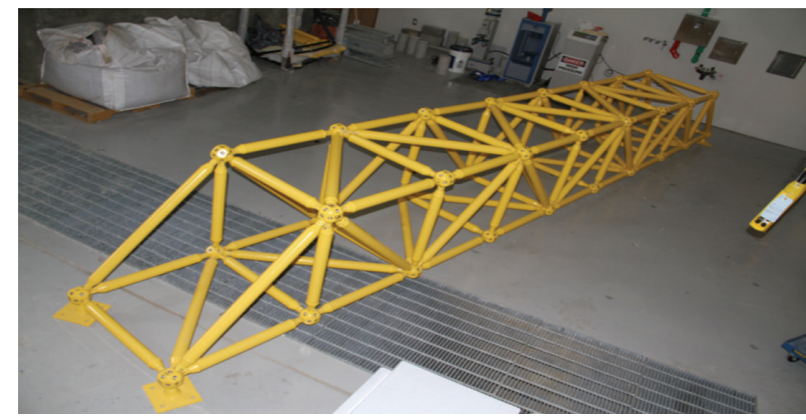

(a) Prototype steel truss structure at the structure laboratory of Jackson State University

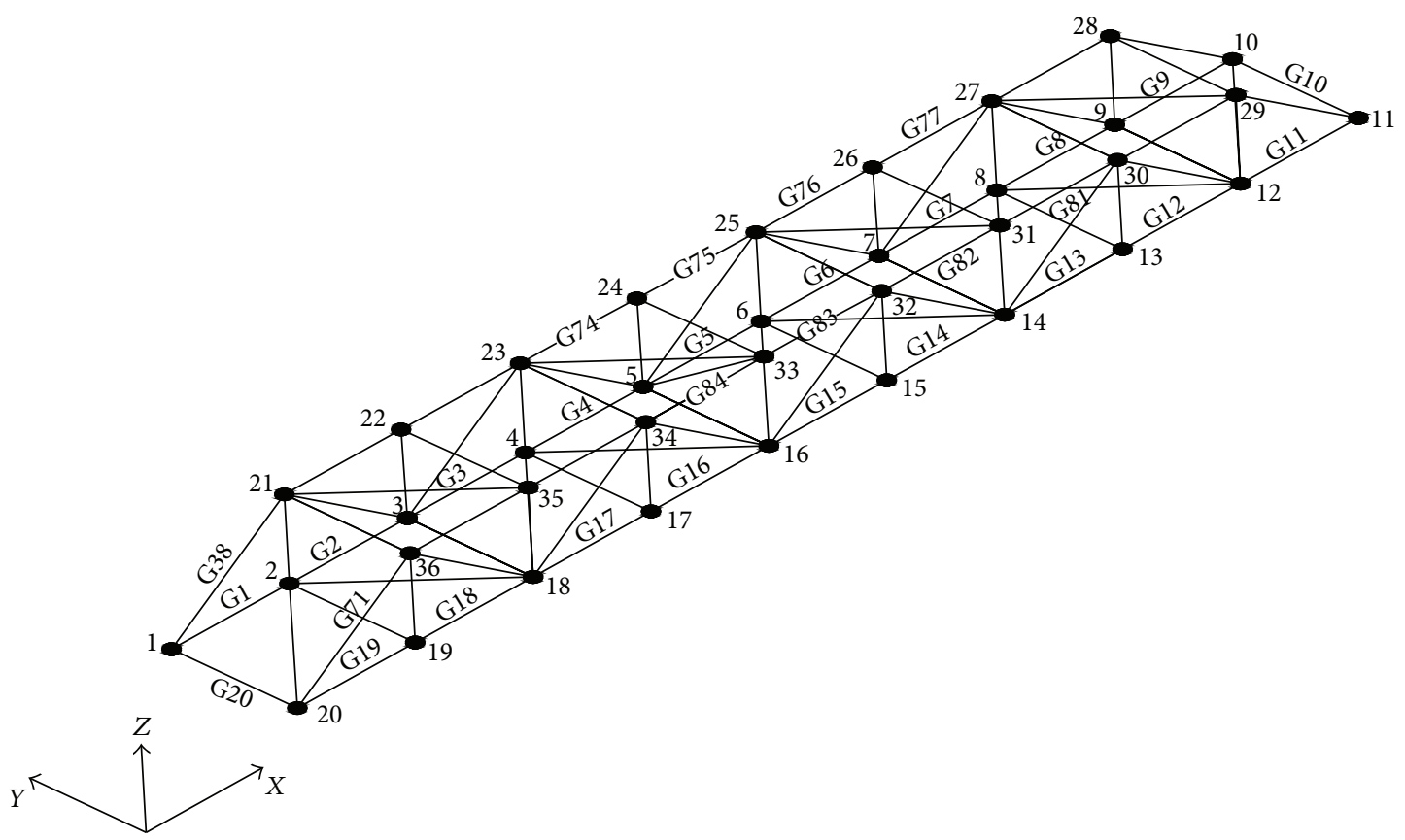

(b) Simulation model in OpenSees

Figure 1: Simulation model of three-dimensional prototype steel truss structure.

\section{Application Example Using Simulated Measurements}

5.1. Simulated Measurements of a Damaged Truss Structure. The applicability of the proposed framework is examined and illustrated for identifying damages of truss structures by using simulated measurements. The prototype truss structure is a nine-bay steel truss structure as shown in Figure 1(a). The truss members are hollow steel tubes with thread rods on both ends. The truss joints are composed of interchangeable balls with threaded holes that can be used to connect truss members as shown in Figure 2(a). Table 1 summarizes the major material and geometrical properties of members of the truss structure. The acceleration time histories at selected points of the truss structure were simulated with a detailed finite element model of the truss structure and used as simulated measurements.

The finite element model for generating simulated measurements was established by using OpenSees program.
TABLE 1: Material and geometrical properties of the truss members.

\begin{tabular}{lccc}
\hline Parameter & $\begin{array}{c}\text { Column-beam } \\
\text { elements }\end{array}$ & Bolts & Balls \\
\hline Young's modulus $\left(\mathrm{N} / \mathrm{m}^{2}\right)$ & $2.10 E 11$ & $2.10 E 11$ & $2.10 E 11$ \\
Area $\left(\mathrm{m}^{2}\right)$ & $3.05 E-4$ & - & - \\
Density $\left(\mathrm{Kg} / \mathrm{m}^{3}\right)$ & 7850 & 7850 & 7850 \\
Mass $(\mathrm{Kg})$ & 2.835 & 0.05 & 3.868 \\
The Poisson ratio & 0.3 & 0.3 & 0.3 \\
Moment of inertia $I_{x x}\left(\mathrm{~m}^{4}\right)$ & $1.31 E-7$ & - & - \\
Moment of inertia $I_{y y}\left(\mathrm{~m}^{4}\right)$ & $1.31 E-7$ & - & - \\
\hline
\end{tabular}

All truss elements are assumed to be linearly elastic. This computational model consisted of 36 nodes and 100 truss elements. In addition, 200 zero-length elements developed in OpenSees program were adopted between the joint and the end of each truss element to simulate the complexity 


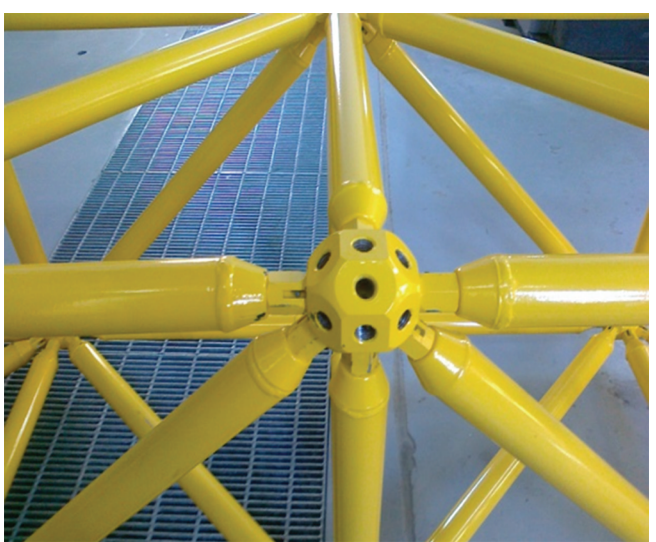

(a) Details of prototype joint

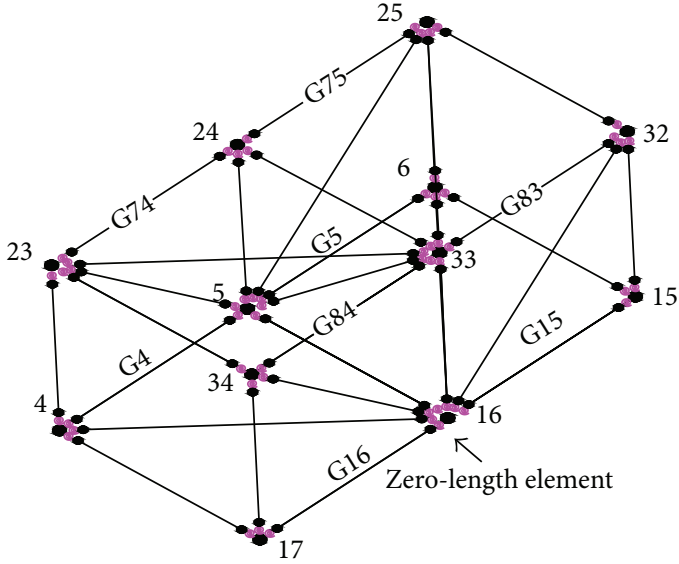

(b) Finite element model of truss joints

Figure 2: Details of prototype joint and finite element model of truss joints.

TABLE 2: Sensor deployment schemes.

\begin{tabular}{|c|c|c|}
\hline $\begin{array}{l}\text { Measurement } \\
\text { scheme }\end{array}$ & Measurement type & $\begin{array}{l}\text { Measurement nodes } \\
\text { (locations) }^{*}\end{array}$ \\
\hline Four-sensor & Vertical acceleration & $5,6,15$, and 16 \\
\hline Eight-sensor & Vertical acceleration & $5,6,7,8,15,16,17$, and 18 \\
\hline Ten-sensor & Vertical acceleration & $\begin{array}{c}5,6,7,8,15,16,17,18,22, \\
\text { and } 31\end{array}$ \\
\hline
\end{tabular}

${ }^{*}$ Node locations can be referred to nodes in Figure 1(b).

of truss joints. The rotational stiffness of the zero-length element is assigned with the value of $9 \times 10^{5} \mathrm{~N} \cdot \mathrm{m} / \mathrm{rad}$ to simulate the joints that are not purely hinged or fixed. This computational model is supported at the four corner bottom joints. Details of the computational model are shown in Figure 1(b). The connection model around the truss joints is shown in Figure 2(b).

The simulated damage of truss structure was represented by the parameters of the stiffness reduction factor $\boldsymbol{\theta}$ of selected truss members. In this simulation, damage is assumed to occur in four truss elements with the element numbers $74,75,83$, and 84 on the top center of the truss structure (see Figure 1(b)). To simulate the damage extents, the stiffness parameters $\boldsymbol{\alpha}$ of the element number 74, 75, 83 and 84 truss elements are, respectively, reduced by $15 \%, 15 \%$, $15 \%$, and $0 \%$, which leads to $\boldsymbol{\alpha}=\{0.85,0.85,0.85,1\}^{T}$.

To obtain simulated vibration measurements of the damaged truss structure, the excitations of the white noises are applied equally at the four top joints of the truss structure in the vertical direction. To examine the impact of quantities of measurements on the accuracy of damage identification, three different sensor deployment schemes, that is, foursensor measurement, eight-sensor measurement, and tensensor measurement (see Table 2), are simulated. The vertical acceleration responses at selected nodes with duration of 7.5 seconds were taken as the accelerometer measurements. With a time interval of 0.015 seconds, the number of total discrete time history data is $N_{t}=500$. To simulate sensor measurement errors, a white noise with $10 \%$ of the noise-tosign ratio (in the root mean square) is added to the simulated measurement data of acceleration.

\subsection{Probabilistic Inference of Truss Member Damage under} Uncertainties of Joint Model. The proposed computational framework and its effectiveness are illustrated and examined through its application for damage identification of the truss structure using simulated accelerometer measurements as described above. For addressing uncertainties associated with the joint model, five different competing model classes were considered and compared for their accuracy in identifying truss member damages. For simplicity in the illustration, the selected five candidate model classes differ from each other only in modeling truss joints and are described in Table 3. Among these five competing model classes, the model class M3 is the same model as that used to generate the simulated acceleration measurement as described in the above section. Thus, this model class M3 represents a model class with no model uncertainties. The model class M1 and model class M2 are simplified models by using either the hinged or the fixed joint model. Both the model M4 and the model M5 modeled the complexity of joint configurations but lack the accuracy of joint property, either underestimating or overestimating the joint rotational stiffness of the model M4 and model M5. Thus, those competing model classes represent uncertainties associated with the model of the truss structure.

The simulated measurements from different sensor deployment schemes (see Table 1) were used as input data for damage identification through proposed probabilistic computational framework. For each given model class, the uncertain stiffness reduction factor vector $\boldsymbol{\alpha}$ was assigned to the potentially damaged structural members to parameterize the extents and locations of damages in the truss structure. Besides, there is another uncertain parameter of the standard deviation $\sigma$ of the model prediction error. Thus, totally five uncertain parameters are needed to be updated using available measurements. Based on prior knowledge and 
TABLE 3: Five competing model classes used for damage identification.

\begin{tabular}{ll}
\hline Model & Model description \\
\hline M1 & Simple spatial truss structure model with fully pinned/hinge joints \\
M2 & Simple spatial truss structure model but with rigid joint connections \\
M3 & The segment connecting truss members and joints steel ball are represented by zero-length elements \\
& in OpenSees. The rotational stiffness of the zero-length elements is set equal to $9 \times 10^{5} \mathrm{~N} \cdot \mathrm{m} / \mathrm{rad}$ \\
M4 & The same model as model class 3 but with the rotational stiffness of $4.5 \times 10^{5} \mathrm{~N} \cdot \mathrm{m} / \mathrm{rad}$ \\
M5 & The same model as model class 3 but with the rotational stiffness of $13.5 \times 10^{5} \mathrm{~N} \cdot \mathrm{m} / \mathrm{rad}$ \\
\hline
\end{tabular}

TABLE 4: Identified damages based on model class M3 with three measurement schemes.

\begin{tabular}{|c|c|c|c|c|c|}
\hline \multirow[b]{2}{*}{$\begin{array}{l}\text { Number of sensor } \\
\text { measurements }\end{array}$} & \multicolumn{4}{|c|}{ Damage index $\boldsymbol{\theta}_{i}$ of different truss members and its $\mathrm{COV}^{\mathrm{b}}$} & \multirow{2}{*}{$\begin{array}{l}\text { Standard deviation of } \\
\text { prediction error } \sigma^{2}(\mathrm{COV})\end{array}$} \\
\hline & $\begin{array}{c}\alpha_{1}(\mathrm{COV}) \\
\text { Member G74 }\end{array}$ & $\begin{array}{c}\alpha_{2}(\mathrm{COV}) \\
\text { Member G75 }\end{array}$ & $\begin{array}{c}\alpha_{3}(\mathrm{COV}) \\
\text { Member G83 }\end{array}$ & $\begin{array}{c}\alpha_{4}(\mathrm{COV}) \\
\text { Member G84 }\end{array}$ & \\
\hline $\begin{array}{l}\text { 4-sensor } \\
\text { (nodes 5-8) }\end{array}$ & $\begin{array}{c}0.8880 \\
(11.189 \%)\end{array}$ & $\begin{array}{c}0.9202 \\
(16.211 \%)\end{array}$ & $\begin{array}{c}0.8612 \\
(11.318 \%)\end{array}$ & $\begin{array}{c}0.9075 \\
(9.6544 \%)\end{array}$ & $\begin{array}{c}47.861 \\
(1.0072 \%)\end{array}$ \\
\hline $\begin{array}{l}\text { 8-sensor } \\
\text { (nodes 5-8,15-18) }\end{array}$ & $\begin{array}{c}0.8938 \\
(10.827 \%)\end{array}$ & $\begin{array}{c}0.8263 \\
(10.120 \%)\end{array}$ & $\begin{array}{c}0.8884 \\
(9.785 \%)\end{array}$ & $\begin{array}{c}0.9250 \\
(8.692 \%)\end{array}$ & $\begin{array}{c}56.838 \\
(1.016 \%)\end{array}$ \\
\hline $\begin{array}{l}10 \text {-sensor } \\
(5-8,15-18,22 \text {, and } 31)\end{array}$ & $\begin{array}{c}0.8370 \\
(7.1801 \%)\end{array}$ & $\begin{array}{c}0.8527 \\
(7.3334 \%)\end{array}$ & $\begin{array}{c}0.8602 \\
(8.3060 \%)\end{array}$ & $\begin{array}{c}0.9507 \\
(5.8878 \%)\end{array}$ & $\begin{array}{c}37.886 \\
(0.4888 \%)\end{array}$ \\
\hline True value & 0.85 & 0.85 & 0.85 & 1.00 & - \\
\hline
\end{tabular}

Note: a: the measure point is referred to as sensor deployment schemes in Table 2.

$\mathrm{b}$ : COV is the coefficient of variation of identified damage index and is listed in parenthesis below.

engineering judgment, the prior PDFs of these four unknown parameters of stiffness reduction factors are assumed to be independently and uniformly distributed between 0.7 and 1.05 , while $\sigma^{2}$ is assumed to be uniformly distributed between 0 and 100, which is about one-half of the mean square of the "measured" acceleration time history.

For each model class with given set of measurements, the TMCMC algorithm was run with $N=1000$ samples for each intermediate stage (or level). About 14 stages were needed to obtain the posterior PDF of uncertain parameters from the prior PDF. For vibration measurements of a duration of 7.5 seconds and a time interval of 0.015 seconds, the total number of discrete time history data is $N_{t}=500$. The computational framework was executed in the desktop PC computer with Intel(R) Core(TM) 2 Quad CPU@ 2.40 GHz, $2.96 \mathrm{~GB}$ of RAM. The computational time for completing the damage identification for each model class was around 13 to 18 hours.

5.3. Results from Implementation of Bayesian Inference. The evolution of the TMCMC samples from the prior PDFs to the posterior PDFs for the model class M3 is illustrated in histograms of model parameter samples as shown in Figure 3. It is noted from Figure 3 that the samples from the prior PDFs are approximately uniformly distributed in the model parameter space at the first stage $\left(p_{0}=0\right)$. Through applying Bayesian inference with TMCMC probabilistic simulations, the samples eventually populate well in the high probability region of the posterior PDFs which is close to the true model parameters at the last stage $\left(t_{m}=1\right)$.

The impact of measurement quantities on the accuracy of damage identification is revealed from results of damage identification based on the model class M3 using three different sensor deployments as specified in Table 2. Those results are presented in Table 4 with the damage index in terms of the mean of stiffness factors, $\alpha_{i}(i=1$ to 4 ) for potential damaged members G74, G75, G83, and G84 and their corresponding coefficient of variation (COV), which is defined as the ratio of the standard deviation to the mean of samples of each stiffness factors $\alpha_{i}(i=1$ to 4$)$, as well as the squared standard deviation $\sigma^{2}$ of prediction errors between the measured accelerations and the predicted accelerations and its coefficient of variation (COV). Those results show that the 10 -sensor measurements can achieve more accurate results than 4-sensor measurements in damage identification and indicate that the accuracy as well as confident level of the identified damages and the associated uncertainties, which is defined by difference of the mean value of parameter and true value, as well as their corresponding coefficient of variation (COV), can be improved as the number of deployed sensors increases. Therefore, increasing the number of effectively-deployed sensors can reduce uncertainties of identified damages.

The impacts of model uncertainties on the damage identification are revealed through comparing identified damage obtained from different candidate model classes. Table 5 presents the identified stiffness factor or damage index $\alpha_{i}$ ( $i=1$ to 4 ) of potential damaged members obtained from five different competing model classes with measurements from two sensor deployment schemes: 4-sensor measurements and 10 -sensor measurements. It reveals that for all five different competing model classes, the accuracy of the identified damage indexes $\alpha_{i}(i=1$ to 4 ) is improved, and the corresponding uncertainties of those damage indexes (represented by VOC) are reduced as the number of deployed sensors increases. 

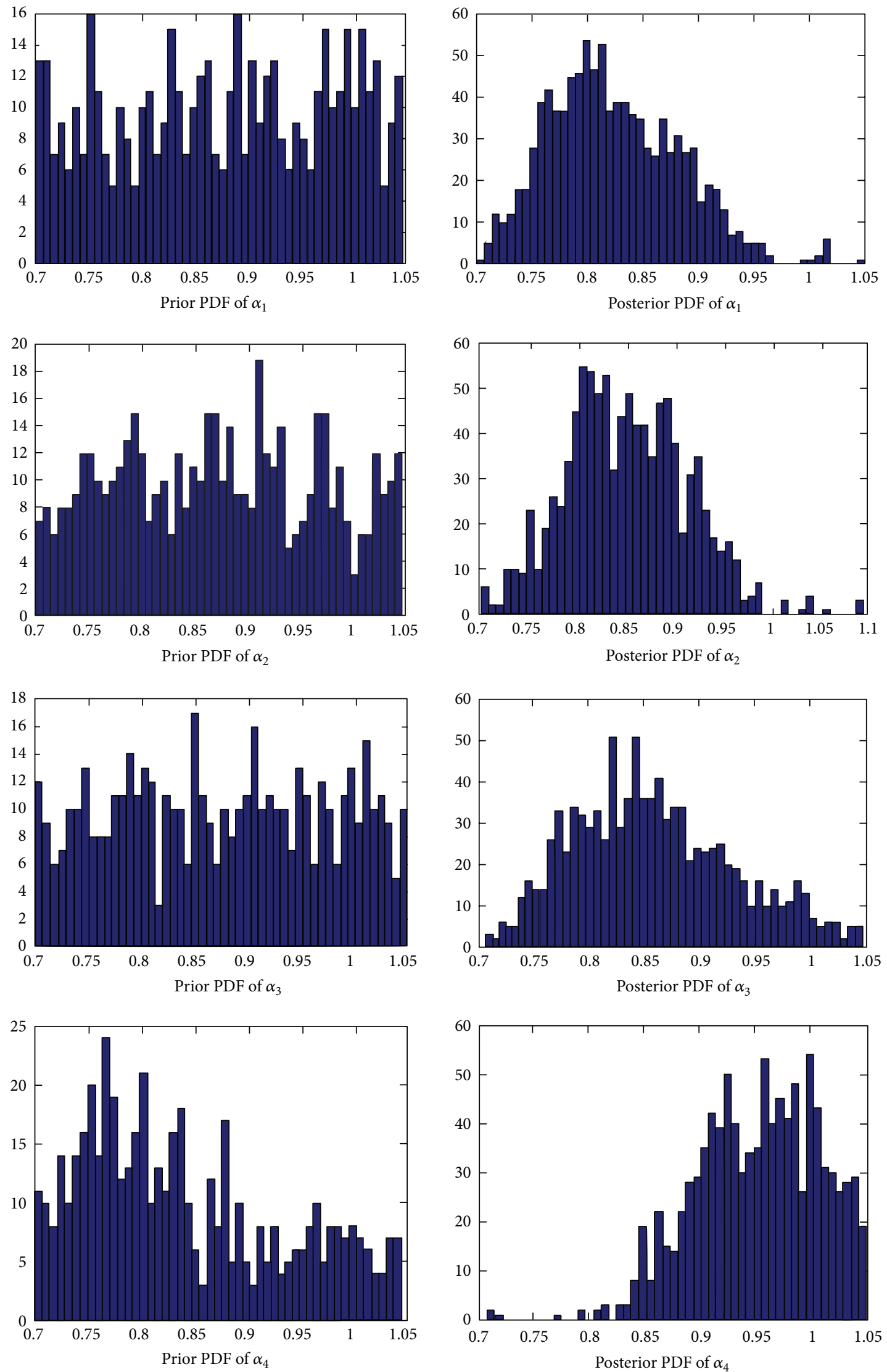

Figure 3: Histograms of PDFs of parameters from $\alpha_{1}$ to $\alpha_{4}$ for the model M3. 
TABLE 5: Identified damages based on five model classes with two measurement schemes.

\begin{tabular}{|c|c|c|c|c|c|c|c|}
\hline \multirow[b]{2}{*}{ Model class } & \multirow[b]{2}{*}{ Damage parameter } & \multicolumn{3}{|c|}{ Using data from 4 measure points } & \multicolumn{3}{|c|}{ Using data from 10 measure points } \\
\hline & & $\begin{array}{l}\text { Identified value } \\
\text { (actual value) }\end{array}$ & $\mathrm{COV}$ & $\log$ evidence $^{*}$ & $\begin{array}{l}\text { Identified value } \\
\text { (actual value) }\end{array}$ & $\mathrm{COV}$ & $\log$ evidence* \\
\hline \multirow{4}{*}{ M1 } & $\alpha_{1}$ & $0.9168(0.85)$ & $8.226 \%$ & \multirow{4}{*}{-2139.6} & $0.8115(0.85)$ & $5.040 \%$ & \multirow{4}{*}{-4560.6} \\
\hline & $\alpha_{2}$ & $0.9540(0.85)$ & $8.327 \%$ & & $0.8378(0.85)$ & $7.626 \%$ & \\
\hline & $\alpha_{3}$ & $0.9128(0.85)$ & $9.896 \%$ & & $0.7924(0.85)$ & $7.669 \%$ & \\
\hline & $\alpha_{4}$ & $0.8972(1.0)$ & $9.042 \%$ & & $0.8373(1.0)$ & $10.032 \%$ & \\
\hline \multirow{4}{*}{ M2 } & $\alpha_{1}$ & $0.7688(0.85)$ & $8.4173 \%$ & \multirow{4}{*}{-1903.8} & $0.8266(0.85)$ & $5.707 \%$ & \multirow{4}{*}{-1723.3} \\
\hline & $\alpha_{2}$ & $0.9961(0.85)$ & $10.508 \%$ & & $0.8433(0.85)$ & $6.189 \%$ & \\
\hline & $\alpha_{3}$ & $0.8469(0.85)$ & $5.8919 \%$ & & $0.9071(0.85)$ & $7.752 \%$ & \\
\hline & $\alpha_{4}$ & $0.9504(1.0)$ & $6.3131 \%$ & & $0.9555(1.0)$ & $5.824 \%$ & \\
\hline \multirow{4}{*}{ M3 } & $\alpha_{1}$ & $0.8880(0.85)$ & $11.189 \%$ & \multirow{4}{*}{-1760.5} & $0.8370(0.85)$ & $7.181 \%$ & \multirow{4}{*}{-1361.5} \\
\hline & $\alpha_{2}$ & $0.9202(0.85)$ & $16.211 \%$ & & $0.8527(0.85)$ & $6.662 \%$ & \\
\hline & $\alpha_{3}$ & $0.8612(0.85)$ & $11.318 \%$ & & $0.8602(0.85)$ & $8.306 \%$ & \\
\hline & $\alpha_{4}$ & $0.9075(1.0)$ & $9.654 \%$ & & $0.9507(1.0)$ & $5.887 \%$ & \\
\hline \multirow{4}{*}{ M4 } & $\alpha_{1}$ & $0.8959(0.85)$ & $6.7123 \%$ & \multirow{4}{*}{-1752.5} & $0.8954(0.85)$ & $6.662 \%$ & \multirow{4}{*}{-1543.6} \\
\hline & $\alpha_{2}$ & $0.8313(0.85)$ & $10.538 \%$ & & $0.8729(0.85)$ & $9.782 \%$ & \\
\hline & $\alpha_{3}$ & $0.9340(0.85)$ & $8.1471 \%$ & & $0.8848(0.85)$ & $5.845 \%$ & \\
\hline & $\alpha_{4}$ & $0.8833(1.0)$ & $6.2183 \%$ & & $0.9320(1.0)$ & $8.620 \%$ & \\
\hline \multirow{4}{*}{ M5 } & $\alpha_{1}$ & $0.8221(0.85)$ & $9.107 \%$ & \multirow{4}{*}{-1760.1} & $0.8744(0.85)$ & $8.540 \%$ & \multirow{4}{*}{-1375.7} \\
\hline & $\alpha_{2}$ & $0.8452(0.85)$ & $8.291 \%$ & & $0.8779(0.85)$ & $7.072 \%$ & \\
\hline & $\alpha_{3}$ & $0.9061(0.85)$ & $5.788 \%$ & & $0.8597(0.85)$ & $7.188 \%$ & \\
\hline & $\alpha_{4}$ & $0.9537(1.0)$ & $6.637 \%$ & & $0.9333(1.0)$ & $8.371 \%$ & \\
\hline
\end{tabular}

Note: ${ }^{*} \log$ evidence here refers to the logarithm of the model evidence as defined in (5).

Table 5 also reveals the model evidence of each competing model class in terms of logarithm of the model evidence. With 10 -sensor measurements, the model class M3 has the largest model evidence and presents identified damage index more accurately. This should be apparent because the model M3 was used to generate simulated measurements and has no uncertainties associated with the model. However, when the 4-sensor measurements are used for damage identification, the model class M4, which actually is an inaccurate model, demonstrates the largest model evidence. This could be caused by inaccurate measurements, which were simulated by adding measurement noise. It should be noted that the solutions in Table 5 are based on mean value of statistic samples of unknown model parameters from one probabilistic simulation. Solutions from different probabilistic simulations may be different from each other. However, stability of those solutions will comply with the law of large numbers (LLN). According to the law, the average of the results obtained from a large number of trials should be close to the expected value and will tend to become closer as more trials are performed.

With 10 -sensor measurements, the identified damages by using the model class M3 have the most accuracy, while the identified damages by using the model M1 have the poorest accuracy. This is because the model class M3 does not have model uncertainties and the model class M1 did not consider any extent of rigidity of joints by assuming that joints are simply hinged. While other model classes approximate the joint rigidity, they give the identified damage index close to the simulated damages. While the model classes M2, M4, and
M5 have different model uncertainties, the damage indexes identified from those models are comparable. The accuracy of identified damages obtained from five candidate model classes by using 10-sensor measurement scheme coincides with the actual extent of model uncertainties among five candidate model classes.

The model uncertainties among five candidate model classes and the accuracy of identified damage index $\boldsymbol{\theta}_{i}$ ( $i=1$ to 4 ) from five candidate model classes described above can be easily evaluated in terms of model evidence as defined in (5). The logarithm (log) of model evidence for each competing model class is obtained during TMCMC simulation process and tabulated in Table 5. With 10-sensor measurements, the rank of model classes with larger model evidence is M3, M5, M4, M2, and M1. This rank coincides with the rank of model classes with less model uncertainties. This indicates that the model evidence can be effectively used as an index of model accuracy in terms of model uncertainties. With data from four-sensor measurement, however, the rank of log of model evidence based on results tabulated in Table 5 does not coincide with the rank of model with less model uncertainties. This deviation of the rank of model evidence from the rank of model with less model uncertainties may be attribute to less information (4-sensor measurements) taken from the system and may also be due to that the information could be distorted by the measurement errors, which is simulated by adding white noise of $10 \%$ of the noise-to-sign ratio (in the root mean square). 
TABLE 6: Competing model classes and their prior and posterior probabilities.

\begin{tabular}{|c|c|c|c|c|c|c|c|c|c|c|c|c|c|c|}
\hline \multicolumn{5}{|c|}{ Competing model classes } & \multicolumn{5}{|c|}{ Prior model probability } & \multicolumn{5}{|c|}{ Posterior model probability } \\
\hline \multirow[t]{2}{*}{ M1 } & M2 & M3 & M4 & M5 & M1 & M2 & M3 & M4 & M5 & M1 & M2 & M3 & M4 & M5 \\
\hline & & & & & 0.20 & 0.20 & 0.20 & 0.20 & 0.20 & 0.00 & 0.00 & 1.00 & 0.00 & 0.00 \\
\hline \multirow[t]{2}{*}{ M1 } & M2 & & M4 & M5 & M1 & M2 & & M4 & M5 & M1 & M2 & & M4 & M5 \\
\hline & & & & & 0.25 & 0.25 & & 0.25 & 0.25 & 0.00 & 0.00 & & 0.00 & 1.00 \\
\hline \multirow[t]{2}{*}{ M1 } & M2 & & M4 & & M1 & M2 & & M4 & & M1 & M2 & & M4 & \\
\hline & & & & & 0.33 & 0.33 & & 0.33 & & 0.00 & 0.00 & & 1.00 & \\
\hline \multirow[t]{4}{*}{ M1 } & M2 & & & & M1 & M2 & & & & M1 & M2 & & & \\
\hline & & & & & 0.50 & 0.50 & & & & 0.00 & 1.00 & & & \\
\hline & M2 & & M4 & & & M2 & & M4 & & & M2 & & M4 & \\
\hline & & & & & & 0.5 & & 0.5 & & & 0.00 & & 1.00 & \\
\hline
\end{tabular}

It also should be noted that even though the aforementioned model evidence provides effective index for selecting better models, it is only a relative index for comparison of competing models based on the same sets of measurement data. The model evidence may not provide the solid ground for comparing models under the different measurement conditions. For example, Table 5 shows that the log evidence for the model M1 based on data with 4 measuring points is larger than that with 10 measuring points $(-2139.6>-4560.6)$. This could not imply that the model M1 has more accuracy with 4 measuring points than that with 10 measuring points. In this particular case, the higher model evidence of the model M1 with 4 measuring points may be attributed to the less error due to comparison of the data from a fewer measurement points than that with 10 measuring points. Thus, the model evidence can be only used to compare the models with data from the same measuring points. In general, however, the competing model with higher model evidence based on data from more measurement points may have more accuracy in prediction than the model with lower model evidence based on data from less measurement points.

With model evidence available from TMCMC, the relative probability of model can be evaluated based on (6). The model probability describes the model probability distribution relatively among the selected competing model classes. Thus, the competing candidate model classes must be defined first. The prior model probability among those selected candidate model classes usually can be assumed based on the prior knowledge and engineering judgment. Without any specific information on those selected candidate model classes, the uniformly distributed model probability can be taken; that is, for model classes $M_{i}\left(i=1,2, \ldots, N_{M}\right)$, the prior model probability of each model class can be assumed to be equal to $1 / N_{M}$. Then, the posterior model probability can be calculated based on (6).

When five competing model classes M1, M2, ., M5 are considered as candidate model classes, Table 6 presents the prior model probability and posterior model probability based on the data from 10 measuring points. The prior model probability for each of five model classes can be assumed to be 0.2. After applying Bayes' theorem, the posterior model probability of the model class M3 is 1.0, and the others are near to 0.00 . This indicates the model class M3 is the best model class or the model class with the least model uncertainties among the selected five candidate model classes. This complies with the simulated scenario, in which the model class M3 is the model used to produce the simulated measurements. Thus, the model class M3 theoretically does not have any model uncertainties when it is used for the damage identification through model updating.

When four model classes M1, M2, M4, and M5 are considered without the model class M3, the prior model probability for each of the four model classes can be assumed to be 0.25 , the posterior model probability of the model class M5 is near to 1.00 , and the others are close to 0.00 . The truss joints in the model M5 were modeled as the semirigid joint with the joint rotation stiffness that is larger than its true value that was used in the model class M3 to generate simulated measurements. Even though the model class M4 considers the semi-rigid joint with rotation stiffness that is half of the true value, its model probability is near to 0.00 . This may be because the model class M5 has much better performance in modeling the truss structure than the model class M4.

However, when considering three model classes M1, M2, and M4 with absence of the models M3 and M5, the prior model probability for each of three model classes is 0.33 , the posterior probability of model class M4 is equal to 1.00, and the others are close to 0.00 (see Table 6). This is consistent with the fact that the model class M4 considers the joint as semi-rigid and has a closer representation of the "true" truss structure, while the model classes M1 and M2 simplify the joint model as either hinged or fixed.

If only two model classes M1 and M2 are considered and compared, the prior model probability for each of two model classes is 0.50 , and the posterior probability of the model class M2 is 1.0 (see Table 6). This is consistent with the fact that the model class M2 considers the rigidity of the joint and is closer to the "true" truss structure represented by the model class M3 than the model class M1, whose joints were modeled as hinges. If the candidate model classes only contain model classes M2 and M4, the model probability of the model class M4 is 1.00, which indicates that the model class M4 has much better representation of the "true" truss structure than the model class M2. This is because the model class M4 considers the semirigidity of the joint condition while the model class $\mathrm{M} 2$ has full rigid joint model. 
In summary, the above application example reveals that the model evidence obtained from Bayesian model updating and the model probability obtained from Bayesian model class selection can be effectively used to identify the model which is most close to the "true" truss structure, when there are uncertainties associated with truss joint model and multiple competing candidate model classes are considered. When the true model class is not among the candidate model classes, the joint model that is more rigid than the "true" truss structure usually yields lager model probability than the joint model which is less rigid than the "true" truss structure. The accuracy of identified damage index may be poor if limited measurement data are available. However, it can be improved by increasing sensor channels. For all cases examined in the application example, the model with the largest model probability always much outperforms other candidate model classes. As a result, the model probability of the most plausible model class is near to 1.0, while the probability of other model classes is almost 0.0. In such cases, if Bayesian model averaging is conducted, the model averaging is actually predominated only by the most plausible model class.

\section{Limitation of This Study and Future Research Directions}

This paper explores the applicability of proposed probabilistic framework into damage identification of truss structures under model uncertainties associated with truss joint details and measurement noises. However, the measurement data are numerical simulations obtained from structural finite element model with simulated damages of a truss structure. The complexity of joint configurations of the "true" truss structure is only represented by the semirigid rotational stiffness. The potentially damaged truss members are limited to four members. The further examination should be conducted using the measurement data obtained from the real physical truss structure with more induced damages occurring not only in truss members but also in truss joints. To make the probabilistic computational framework applicable for field implementation, the research needs and directions in this area should focus on two major aspects: one is to improve the computational efficiency of framework for reducing the computational time for implementation of the Bayesian probabilistic framework with more uncertain model parameters and the other is to address the impacts of environmental variations, such as change of temperature or humidity, on structural dynamic responses and distinguish them from those caused by structural damages.

\section{Summary and Conclusion Remarks}

This paper proposes and illustrates a new perspective for vibration-based damage identification of truss structures under model uncertainties associated with truss joints based on Bayesian model updating and model class selection. The proposed probabilistic framework integrates the advanced sampling algorithm of transitional Markov chain Monte
Carlo (TMCMC) with the finite element model created in OpenSees program to derive the probabilistic characteristics of associated uncertainties in terms of statistical samples of parameters of interest, model evidence, and model probabilities of competing model classes. The effectiveness of the framework is examined by using simulated measurements. The simulation results demonstrate that the model evidence obtained from TMCMC can effectively be used to quantify model probabilities of competing candidate model classes for assessing model uncertainties associated with truss joints, while statistical samples drawn from TMCMC can well approximate the updated probabilistic distributions of uncertain model parameters that represent damage in truss structures. It is also indicated that the model probability can provide effective index for describing the relative plausibility of the model class and selecting the model with fewer uncertainties among competing candidate model classes. The proposed framework can be used to identify the probabilistic characteristics of damage of truss members under the joint model uncertainties and measurement noise. The accuracy of identified damage index can be improved by using measurement data from more sensors.

Further examination of the effectiveness of the proposed framework should be conducted by using measurement data obtained from real physical truss structure with more damages occurring not only in truss members but also in truss joints. To make the probabilistic framework ready for practical implementation, the future research should focus on developing efficient algorithms for reducing the high computational demands of probabilistic simulation of large structures and distinguishing the impacts of environmental variations from those caused by damages on the measured structural dynamic response. The research direction in this regard should include (1) adopting efficient gradient-based sampling techniques that can replace random walking algorithm for proposing statistical samples; (2) implementing spectra-based Bayesian inference of damage identification based on output-only dynamic response under ambient vibrations; (3) developing effective surrogate model or reduced model to represent the computationally-expensive structure model; and (4) developing effective dynamic features that are extracted from the sensor measurements and are sensitive to structural damage and insensitive to environment variations for damage identification.

\section{Acknowledgments}

The authors gratefully acknowledge any support from the Institute for Multimodal Transportation at Jackson State University under Grant DTRT06-G-0049 and from National Science Foundation under Award NSF/DUE-0837395. Any opinions, conclusions, or recommendations expressed in this paper are those of the authors and do not necessarily reflect the views of these funding agencies. The authors also thank Professor Jianye Ching from National Taiwan University for providing MATLAB codes of transitional Markov chain Monte Carlo simulation algorithm. 


\section{References}

[1] Y. Gao, B. F. Spencer Jr., and M. Ruiz-Sandoval, "Distributed computing strategy for structural health monitoring," Structural Control and Health Monitoring, vol. 13, no. 1, pp. 488-507, 2006.

[2] Y. Gao and B. F. Spencer Jr., "Online damage diagnosis for civil infrastructure employing a flexibility-based approach," Smart Materials and Structures, vol. 15, no. 1, pp. 9-19, 2006.

[3] B. F. Spencer, Y. Gao, and G. Yang, "Distributed computing strategy for damage monitoring employing smart sensors," in Proceedings of the 2nd International Conference on Structural Health Monitoring of Intelligent Infrastructure, vol. 1, pp. 35-47, 2005.

[4] X. D. Ji, J. R. Qian, and L. H. Xu, "Damage diagnosis of a twostorey spatial steel braced-frame model," Structural Control and Health Monitoring, vol. 14, no. 8, pp. 1083-1100, 2007.

[5] H. Sohn, A Bayesian probabilistic approach to damage detection for civil structures [Ph.D. thesis], Stanford University, 1998.

[6] S. W. Jones, P. A. Kirby, and D. A. Nethercot, "Columns with semi-rigid joints," Journal of Structural Engineering, vol. 108, no. 2, pp. 361-372, 1982.

[7] C. W. Lewitt, E. Chesson, and W. H. Munse, "Engineering Bulletin of university of Illinois, 500 . Restraint characteristics of flexible riveted and bolted beam-to-column connections," 1969.

[8] Z. X. Li, T. Q. Zhou, T. H. T. Chan, and Y. Yu, "Multi-scale numerical analysis on dynamic response and local damage in long-span bridges," Engineering Structures, vol. 29, no. 7, pp. 1507-1524, 2007.

[9] W. D. Zhu and K. He, "Finite element modeling of structures with L-shaped beams and bolted joints," Journal of Vibration and Acoustics, vol. 133, no. 1, Article ID 011011, 2011.

[10] G. J. Yun, "Modal identification and damage detection for structural health monitoring under ambient vibration environment," in Proceedings of the Structures Congress, pp. 1-10, May 2009.

[11] S. S. Law, T. H. T. Chan, and D. Wu, "Super-element with semirigid joints in model updating," Journal of Sound and Vibration, vol. 239, no. 1, pp. 19-39, 2001.

[12] M. C. Kennedy and A. O'Hagan, "Bayesian calibration of computer models," Journal of the Royal Statistical Society B, vol. 63, no. 3, pp. 425-464, 2001.

[13] K. V. Yuen and L. S. Katafygiotis, "Bayesian time-domain approach for modal updating using ambient data," Probabilistic Engineering Mechanics, vol. 16, no. 3, pp. 219-231, 2001.

[14] J. L. Beck and K. V. Yuen, "Model selection using response measurements: Bayesian probabilistic approach," Journal of Engineering Mechanics, vol. 130, no. 2, pp. 192-203, 2004.

[15] Y. B. Chen and M. Q. Feng, "Structural health monitoring by recursive Bayesian filtering," Journal of Engineering Mechanics, vol. 135, no. 4, pp. 231-242, 2009.

[16] E. Ntotsios, C. Papadimitriou, P. Panetsos, G. Karaiskos, K. Perros, and P. C. Perdikaris, "Bridge health monitoring system based on vibration measurements," Bulletin of Earthquake Engineering, vol. 7, no. 2, pp. 469-483, 2009.

[17] K. V. Yuen, "Recent developments of Bayesian model class selection and applications in civil engineering," Structural Safety, vol. 32, no. 5, pp. 338-346, 2010.

[18] M. Muto and J. L. Beck, "Bayesian updating and model class selection for hysteretic structural models using stochastic simulation," JVC/Journal of Vibration and Control, vol. 14, no. 1-2, pp. 7-34, 2008.
[19] J. Ching and Y. J. Chen, "Transitional Markov chain Monte Carlo method for Bayesian model updating, model class selection, and model averaging," Journal of Engineering Mechanics, vol. 133, no. 7, pp. 816-832, 2007.

[20] S. Chib and E. Greenberg, "Understanding the MetropolisHastings algorithm," The American Statistician, vol. 49, no. 4, pp. 327-335, 1995.

[21] W. M. Bolstad, Understanding Computational Bayesian Statistics, John Wiley \& Sons, Hoboken, NJ, USA, 2010.

[22] J. L. Beck and S. K. Au, "Bayesian updating of structural models and reliability using Markov chain Monte Carlo simulation," Journal of Engineering Mechanics, vol. 128, no. 4, pp. 380-391, 2002.

[23] M. Sadrossadat Zadeh and M. Saiid Saiidi, "Pre-test analytical studies of NEESR-SG 4-span bridge model using OpenSees," Report No. CCEER-07-3, Civil Engineering Department, University of Nevada, Reno, Nev, USA, 2007. 

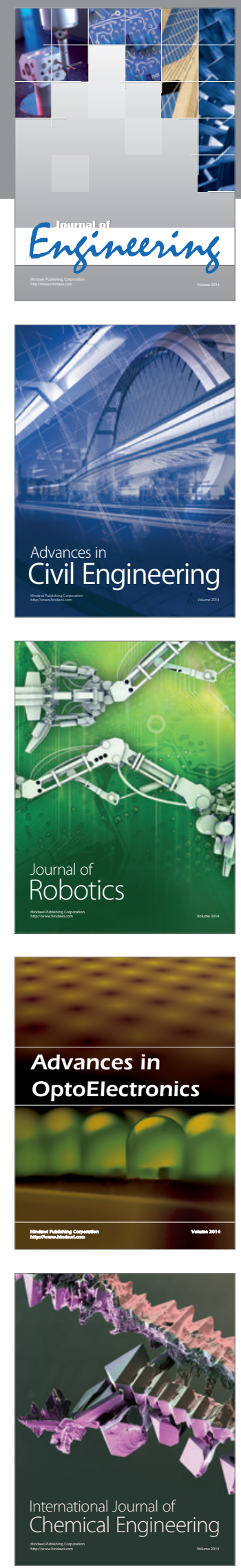

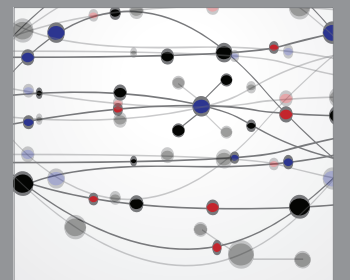

The Scientific World Journal
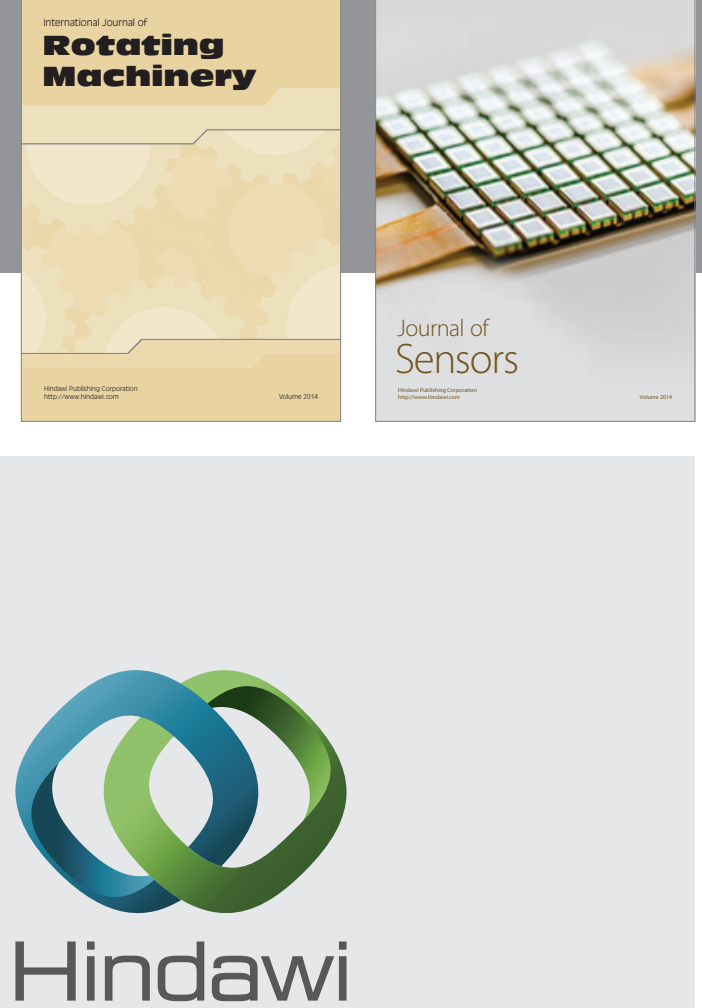

Submit your manuscripts at http://www.hindawi.com
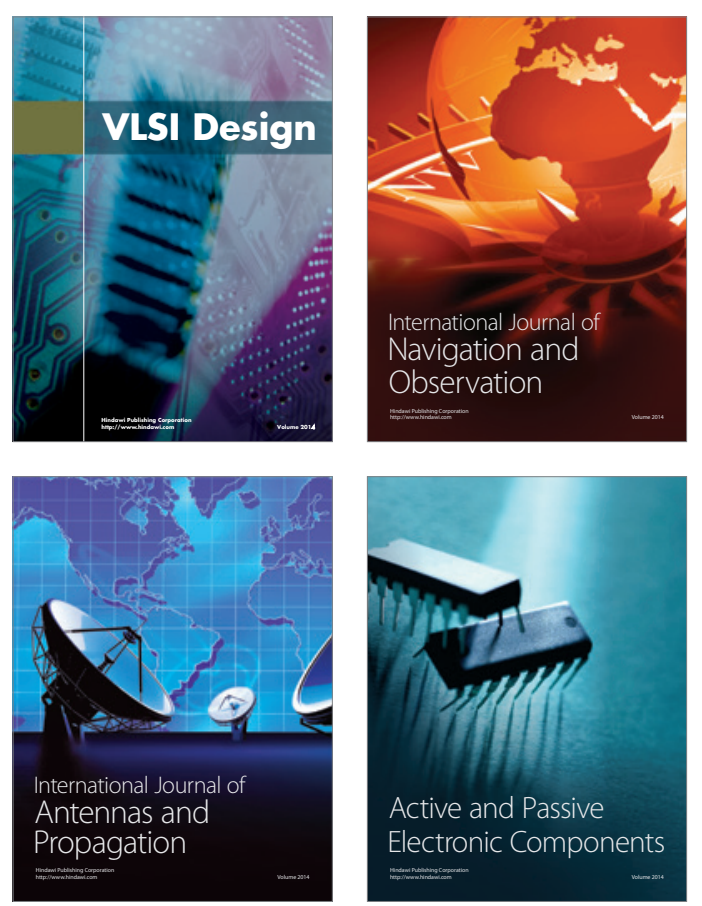
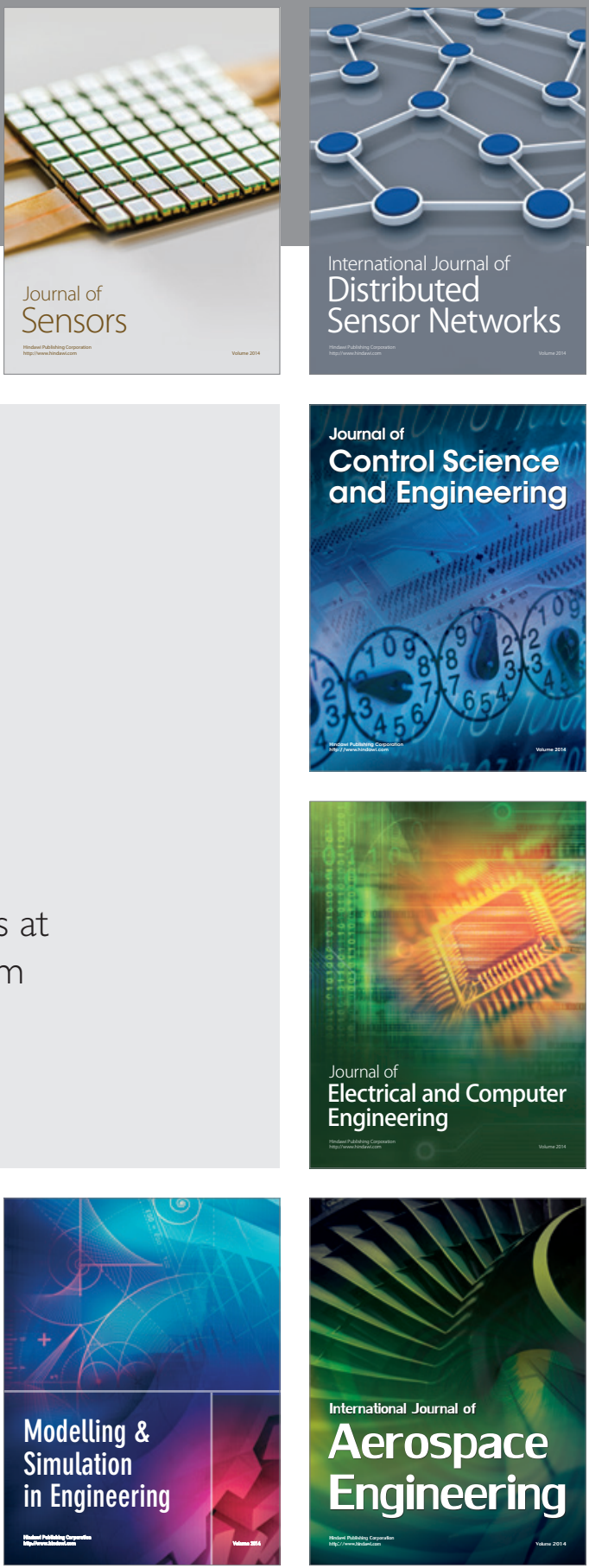

Journal of

Control Science

and Engineering
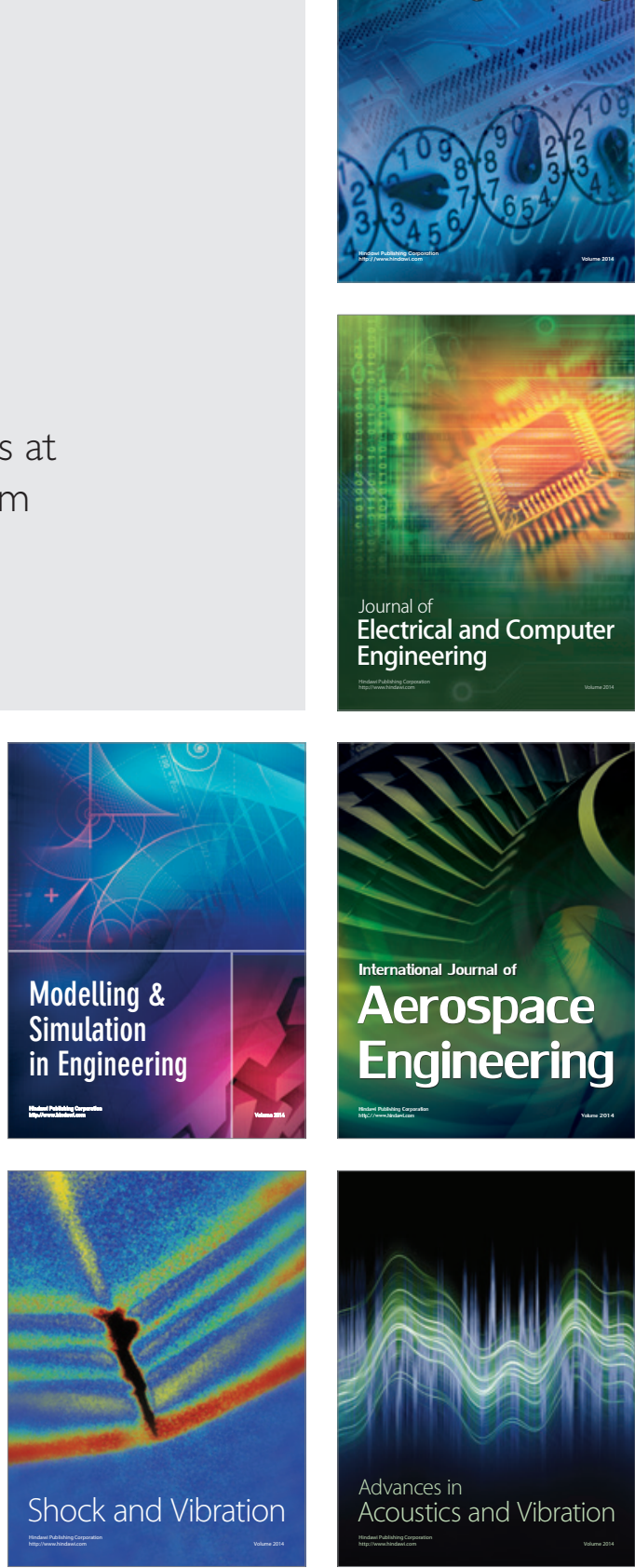